\title{
Expression of deubiquitinases in human gingiva and cultured human gingival
}

\section{fibroblasts}

\author{
Yong-Wei Fu*, Hong-Zhi Xu
}

Department of Stomatology, The First People's Hospital of Lianyungang (Lianyungang

Clinical Medical College of Nanjing Medical University), Lianyungang, China.

*Correspondence: Yong-Wei Fu, Department of Stomatology, The First People's Hospital of Lianyungang, 6 East Zhenhua Road, Haizhou District, Lianyungang 222061, Jiangsu, China.

E-mail: fuyongweivip@ sina.com

Tel.: 086-0518-85767838

Fax: 086-0518-85767213

\section{Acknowledgments}

We are grateful to Yan-Hui Li for her critical reading of the manuscript. 


\title{
Expression of deubiquitinases in human gingiva and cultured human gingival \\ fibroblasts
}

\begin{abstract}
Background: Although deubiquitinating enzymes (DUBs) such as CYLD, A20 and OTULIN are expressed in multiple tissues and thought to be linked with inflammatory diseases, their expression in periodontal tissues remains to be determined. This research was designed to assess the expression of CYLD, A20 and OTULIN in human gingiva, and to evaluate the regulation of these DUBs in human gingival fibroblasts (HGFs) upon different stimuli.
\end{abstract}

Methods: Immunohistochemistry assay was conducted to determine the expression of CYLD, A20 and OTULIN in human gingiva. Immunofluorescence assay was employed to observe the protein expression of CYLD, A20 and OTULIN in HGFs. RT-PCR and western blots were carried out to assess gene and protein expression changes of these DUBs in HGFs upon LPS or TNF- $\alpha$.

Results: CYLD, A20 and OTULIN were found to be expressed in human gingiva and HGFs. Further, the expression of CYLD, A20 and OTULIN in HGFs exhibited distinct regulation by different stimuli.

Conclusions: Our findings suggest that CYLD, A20 and OTULIN might play a role in the progression of periodontitis.

Keywords: CYLD, A20, OTULIN, Gingiva, Fibroblasts 


\section{Background}

Ubiquitin, a protein containing 76 amino acids, has an essential role in variety of biological processes [1-4]. Ubiquitination is a critical post-translational protein modification, performed by specific ubiquitin enzymes [5, 6]. The ubiquitin enzymes attach ubiquitin to target proteins and modulate the function of the target proteins. Attached ubiquitins can be removed by deubiquitinating enzymes (DUBs). Several DUBs including CYLD, A20 and OTULIN are reported to inhibit NF- $\kappa$ B activation and play an important role in modulating immunity and inflammation [7-9]. Furthermore, previous studies have shown that CYLD, A20 and OTULIN are all related to inflammatory diseases [10-13].

Periodontitis is an inflammatory disease of periodontal tissues which is caused by plaque biofilms [14]. In addition to being an important cause of tooth loss in adults, periodontitis is highly linked with multiple systemic diseases [15]. Recent work suggests that dysregulation of immune and inflammatory responses in the periodontium may be involved in the progression of periodontitis $[16,17]$. Briefly, periodontal pathogenic bacteria and their toxic substances interact with host immune cells, result in immune and inflammatory responses in periodontal tissues, trigger the excessive release of inflammatory cytokines, and eventually cause periodontal tissue destruction [18].

Recent work demonstrates that regulating the immune responses may act as an adjunct to traditional periodontal therapy $[19,20]$.

$\mathrm{NF}-\kappa \mathrm{B}$ is an important regulator of immune and inflammatory responses $[8,21]$. Upregulated NF- $\mathrm{kB}$ activity is reported in many inflammatory conditions such as inflammatory bowel disease, rheumatoid arthritis, and periodontitis [22, 23]. Evidence increasingly suggests that NF- $\kappa \mathrm{B}$ activation may be involved in the progression of periodontitis [22, 24]. Periodontal pathogens may promote the induction of 
proinflammatory cytokines in periodontal tissues by upregulating NF- $\kappa \mathrm{B}$ activation [18]. Considering the repressing effect of CYLD, A20 and OTULIN on NF- $\kappa B$ activation, it is reasonable to postulate that these DUBs may restrict periodontal inflammatory responses.

Although CYLD, A20 and OTULIN are expressed in multiple tissues, their expression in periodontal tissues remains to be elucidated. The objective of this research is to assess the expression of CYLD, A20 and OTULIN in human gingiva and to evaluate the regulation of these DUBs in HGFs upon different stimuli.

\section{Methods}

\section{Antibodies and reagents}

Rabbit anti-CYLD (catalog \#ab137524), rabbit anti-A20 (catalog \#ab92324) and rabbit anti-OTULIN (catalog \#ab151117) were purchased from Abcam (Cambridge, UK). Normal rabbit IgG (catalog \#A7016) were purchased from Beyotime Biotechnology (Shanghai, China). Alexa Fluor 568 goat anti-rabbit IgG (catalog \#A11036) was supplied by Thermo Fisher Scientific (Rockford, IL, USA). Tubulin rabbit polyclonal antibody (catalog \#10094-1-AP) and horseradish peroxidase-conjugated secondary antibody (catalog \#SA00001-2) were supplied by Proteintech Group (Wuhan, China). Minimum essential medium alpha was supplied by Thermo Fisher Scientific (Suzhou, China). Penicillin-streptomycin solution (catalog \#SV30010) was supplied by HyClone (Austria). Fetal bovine serum (catalog \#VS500T) was supplied by Ausbian (Australia). Immunohistochemistry application solutions kit (catalog \#13079) was supplied by Cell

Signaling Technologies (Danvers, MA, USA). DAPI (catalog \#R37606) and ProLong

Diamond Antifade Mountant (catalog \#P36965) were supplied by Molecular Probes (Eugene, OR, USA). TNF- $\alpha$ (catalog \#01375) was supplied by R\&D Systems (Minneapolis, MN, USA). LPS (catalog \#tlrl-pglps) was supplied by InvivoGen (San 
Diego, USA). RNA extraction kit (catalog \#R6834-01) was from OMEGA Bio-Tek (Norcross, GA, USA). PrimeScript RT Master Mix kit (catalog \#RR036A) and SYBR Premix Ex Taq $\square$ kit (catalog \#RR820A) were from Takara Bio Inc. (Otsu, Japan). BCA Protein Assay Kit (catalog \#23227) was from Pierce Biotechnology (Rockford, IL, USA).

\section{Gingival samples}

Human gingival biopsy samples were acquired from 12 individuals (5 male and 7 female, average age of 28.7 y) undergoing crown-lengthening surgery. All individuals were selected from the First People's Hospital of Lianyungang between March 2019 and August 2019. Informed consent forms were assigned by all individuals before they were enrolled in the research. This study was approved by The Ethics Committee of Nanjing Medical University (2018389). All experiments on human subjects were conducted in accordance with the 1964 Helsinki declaration and its later amendments or comparable ethical standards.

\section{Immunohistochemistry assay}

Gingival samples were fixed in $4 \%$ paraformaldehyde for $24 \mathrm{~h}$ at $4 \square$. Then these samples were processed to paraffin blocks and sectioned at $5 \mu \mathrm{m}$. Immunohistochemical study was undertaken as previously described using a commercial immunohistochemistry kit [4]. Anti-CYLD (1:400), anti-A20 (1:400) and anti-OTULIN $(1: 400$,$) antibodies were applied in this study. Nonimmunized IgG (1:400, Beyotime$

Biotechnology) was used as negative controls in the present study. Slices were photographed with a Leica DM4000 B microscope.

\section{Cell culture}

HGFs were isolated from human gingival tissues and cultured with an explant culture method. Briefly, gingival tissues were acquired from three healthy individuals (2 
females and 1 male, aged 11-14 y) experiencing first premolar extractions for orthodontic therapy. The extracted teeth were placed in phosphate buffered saline (PBS) and immediately transferred to the laboratory. Then the gingival tissues attached to the cervical region of the extracted teeth were removed and cut into small pieces in the biosafety cabinet. Afterwards, the tissue pieces were transferred to $25 \mathrm{~cm}^{2}$ culture flasks and cultured in minimum essential medium alpha supplemented with $15 \%$ fetal bovine serum. HGFs at passages 3 to 6 were used in this study.

\section{Immunofluorescence staining}

For Immunofluorescence Staining, HGFs were seed in 24-well culture plates with a coverslip at the bottom in a density of $2.5 \times 10^{4}$ cells per well. After $24 \mathrm{~h}$, HGFs were fixed with $4 \%$ paraformaldehyde in PBS for 15 min, permeabilized with $0.2 \%$ Triton X100 in PBS for 5 min, blocked with 1\% BSA in PBS for $1 \mathrm{~h}$ at room temperature (RT). Then HGFs were incubated with primary antibodies against CYLD (1:200), A20 (1:200) and OTULIN (1:200) overnight at 4 $\square$. Afterwards, HGFs were stained with Alexa Fluor 568-conjugated secondary antibody $(4 \mu \mathrm{g} / \mathrm{mL})$ for $50 \mathrm{~min}$ and then with DAPI for 6 min at RT. Each of the above-mentioned steps was followed by three 5-min washes in PBS. Finally, coverslips were mounted using ProLong Diamond Antifade Mountant and data were obtained by using a Leica DM4000 B microscope.

\section{Cell stimulation}

HGFs were seed in 6-well culture plates and cultured for $24 \mathrm{~h}$. After washing twice with PBS, HGFs were starved for $12 \mathrm{~h}$ in $\alpha$-MEM without foetal bovine serum. Then HGFs were stimulated with $1 \mu \mathrm{g} / \mathrm{ml}$ LPS or $10 \mathrm{ng} / \mathrm{ml} \mathrm{TNF-} \alpha$ as indicated.

\section{RNA isolation and reverse transcription polymerase chain reaction (RT-PCR)}

Total RNA was extracted from treated HGFs with an RNA extraction kit per the supplier's protocol. cDNA synthesis was undertaken using a PrimeScript RT Master 
Mix kit. Then RT-PCR was carried out using a SYBR Premix Ex Taq $\square$ kit on a 7300

Real Time PCR System. Each sample was run in triplicate. Results were normalized to

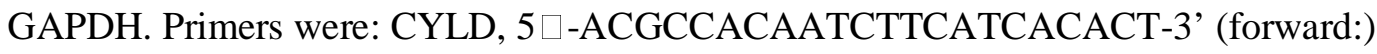
and 5 $\square$-AGGTCGTGGTCAAGGTTTCACT-3' (reverse); ; A20, 5'ATGCACCGATACACACTGGA-3' (forward) and 5'GGATGATCTCCCGAAACTGA-3' (reverse); OTULIN, 5'AAAGAGGGGCATCAGAACCG-3' (forward) and 5'GGCCCTCAGTGCACAGTAAT-3' (reverse); and GAPDH, 5'-

TGCACCACCAACTGCTTAGC-3' (forward) and 5'-GGCATGGACTGTGGTCATGAG-3’ (reverse).

\section{Protein extraction and western blotting}

For western blotting, pre-treated HGFs were lysed using RIPA lysis buffer containing protease inhibitors. The protein concentrations were determined with a BCA Protein Assay Kit. Then, the protein extracts were subjected to western blotting with antibodies to CYLD (1:1000), A20 (1:1000), OTULIN (1:400), and Tubulin (1:1000). Horseradish peroxidase-conjugated secondary antibody (1:8000) was applied. Densitometry quantification of the protein bands was processed with ImageJ software.

\section{Statistical analysis}

Data are expressed as the mean $\pm \mathrm{SD}$. ANOVA followed by Dunnett's multiple comparison test was used to determine the significance of differences. GraphPad Prism 5 was used to perform statistical analyses. Differences were considered to be significant when $P<0.05$.

\section{Results}

\section{Expression of deubiquitinases in human gingival tissues}


medRxiv preprint doi: https://doi.org/10.1101/2021.01.26.21250532; this version posted March 16, 2021. The copyright holder for this preprint (which was not certified by peer review) is the author/funder, who has granted medRxiv a license to display the preprint in perpetuity.

All rights reserved. No reuse allowed without permission.

To determine the expression of CYLD, A20 and OTULIN in human gingiva, we performed immunohistochemistry staining on gingival tissue samples from 12 individuals. Positive staining cells for CYLD, A20and OTULIN were observed both in the epithelium and in the connective tissue (Fig. 1). Further, all of the above deubiquitinases were mainly expressed in epithelial cells.
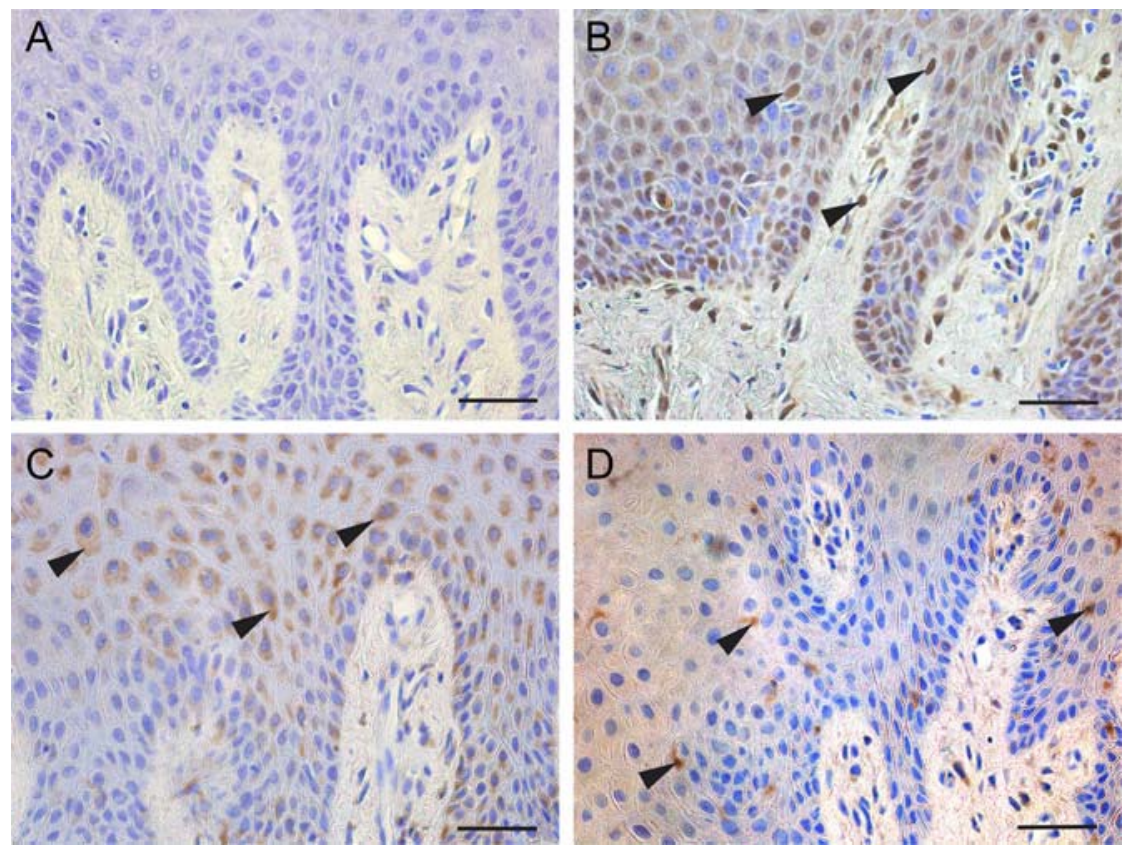

Fig. 1 Immunohistochemistry for CYLD, A20 and OTULIN on sections from gingival tissue. a The negative control of immunohistochemistry. b CYLD staining in gingival tissue. Arrows show positive staining for CYLD. c A20 staining in gingival tissue. Arrows show positive staining for A20. d OTULIN staining in gingival tissue. Arrows show positive staining for OTULIN. Scale bar, $50 \mu \mathrm{m}$

\section{Expression of deubiquitinases in human gingival fibroblasts}

Immunofluorescence was performed to assess the expression of deubiquitinases in HGFs. As expected, HGFs displayed positive staining for CYLD, A20 and OTULIN.

(Fig. 2). 
A

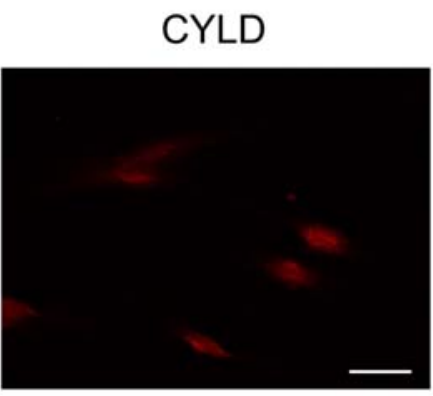

B

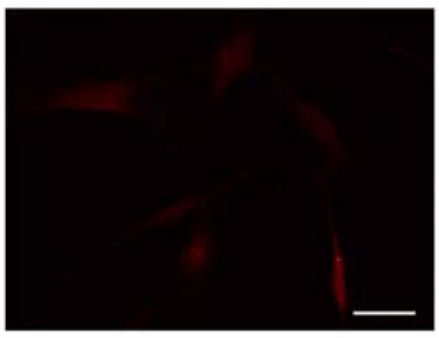

C

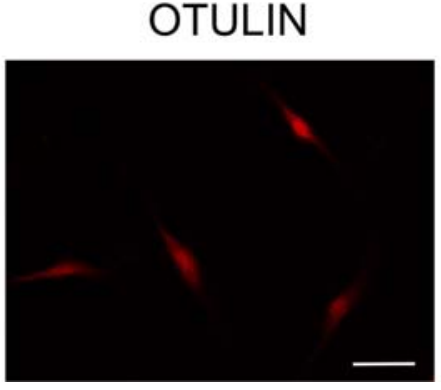

DAPI

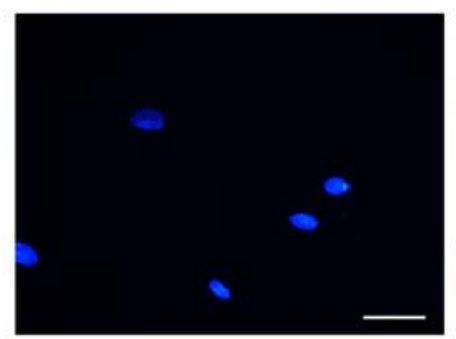

DAPI

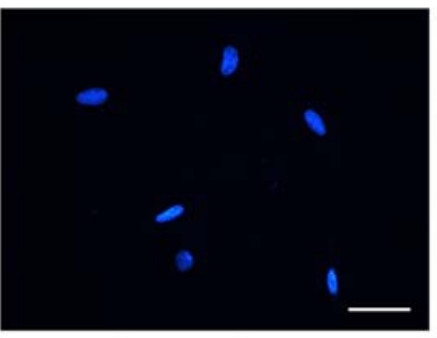

DAPI

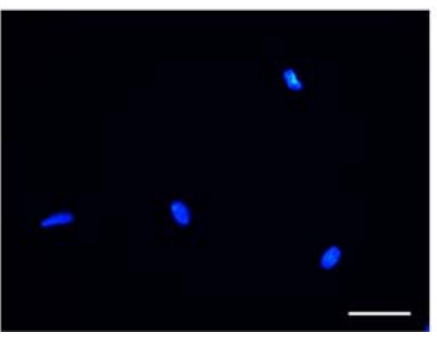

Merge

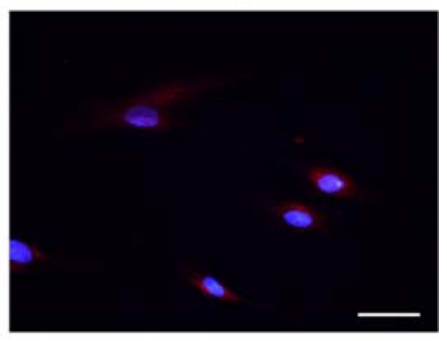

Merge

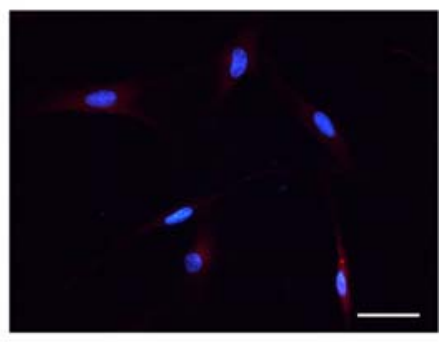

Merge

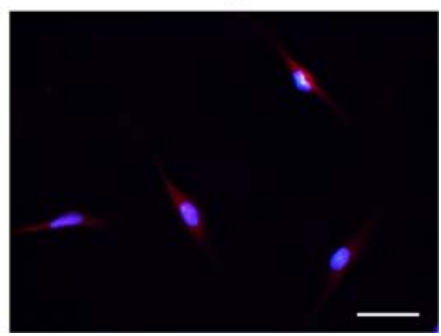

Fig. 2 Immunofluorescence staining for CYLD (a), A20 (b) and OTULIN (c) in HGFs. Scale bar, $50 \mu \mathrm{m}$

\section{Regulation of deubiquitinases by different stimuli}

To examine the changes in the expression levels of deubiquitinases in LPS- or TNF- $\alpha$ treated HGFs, RT-PCR and western blotting were performed. Results demonstrated that the expression of CYLD, A20 and OTULIN in HGFs exhibits distinct regulation upon LPS or TNF- $\alpha$ (Fig. 3 and Fig. 4).

The RT-PCR results demonstrated that the CYLD mRNA level had no obvious change after treatment with LPS or TNF- $\alpha$ (Fig. 3a and 3d). Intriguingly, the mRNA 
expression of A20 was decreased $1 \mathrm{~h}$ after treatment with LPS, and returned to the baseline level after $5 \mathrm{~h}$ (Fig. 3b). As expected, the mRNA expression of A20 was rapidly upregulated in response to TNF- $\alpha$ (Fig. 3e). In addition, LPS led to an increase in OTULIN mRNA expression after $3 \mathrm{~h}$ (Fig. $3 \mathrm{c}$ ). In contrast, TNF- $\alpha$ resulted in a decrease in the mRNA expression of OTULIN after $1 \mathrm{~h}$ (Fig. 3f).

At the protein level, CYLD expression had no significant change upon LPS or TNF- $\alpha$ treatment (Fig. 4a and 4b). In addition, the protein expression of A20 markedly increased after treatment with LPS or TNF- $\alpha$ (Fig. 4a and 4b). Furthermore, LPS also led to the increase of OTULIN protein expression (Fig. 4a). However, TNF- $\alpha$ treatment did not affect the protein expression of OTULIN (Fig. 4b).

A
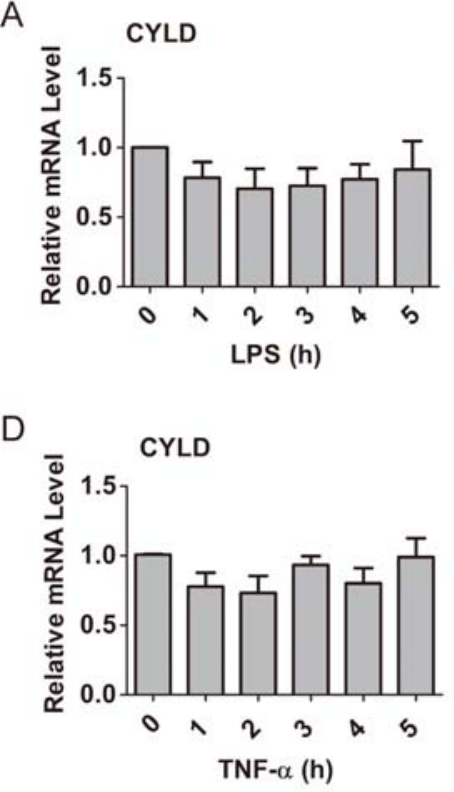

B

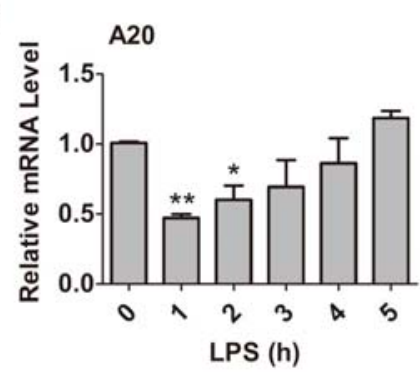

E

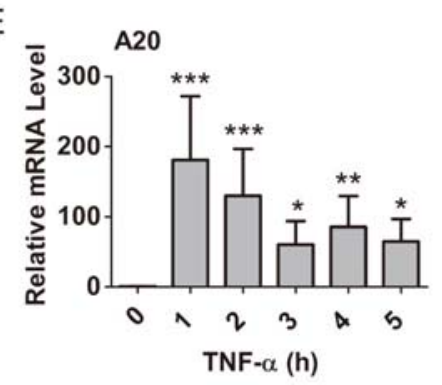

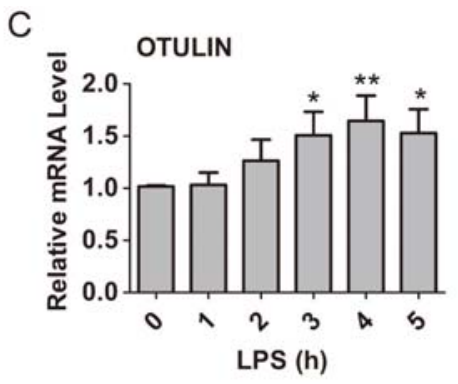

$\mathrm{F}$

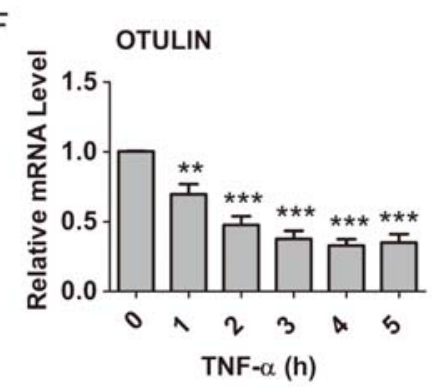

Fig. 3 mRNA levels of CYLD, A20 and OTULIN in HGFs pretreated with LPS $(\mathbf{a}, \mathbf{b}, \mathbf{c})$ or TNF- $\square(\mathbf{d}, \mathbf{e}, \mathbf{f})$ for the indicated periods of time. ${ }^{\square} P<0.05$, ${ }^{0} P<0.01$, ${ }^{0}{ }^{0} P<0.001,0$ $\mathrm{h}$ versus $1 \mathrm{~h}, 2 \mathrm{~h}, 3 \mathrm{~h}, 4 \mathrm{~h}$ or $5 \mathrm{~h}$, one-way ANOVA with Dunnett's multiple comparison test. Data presented are from three independent experiments 

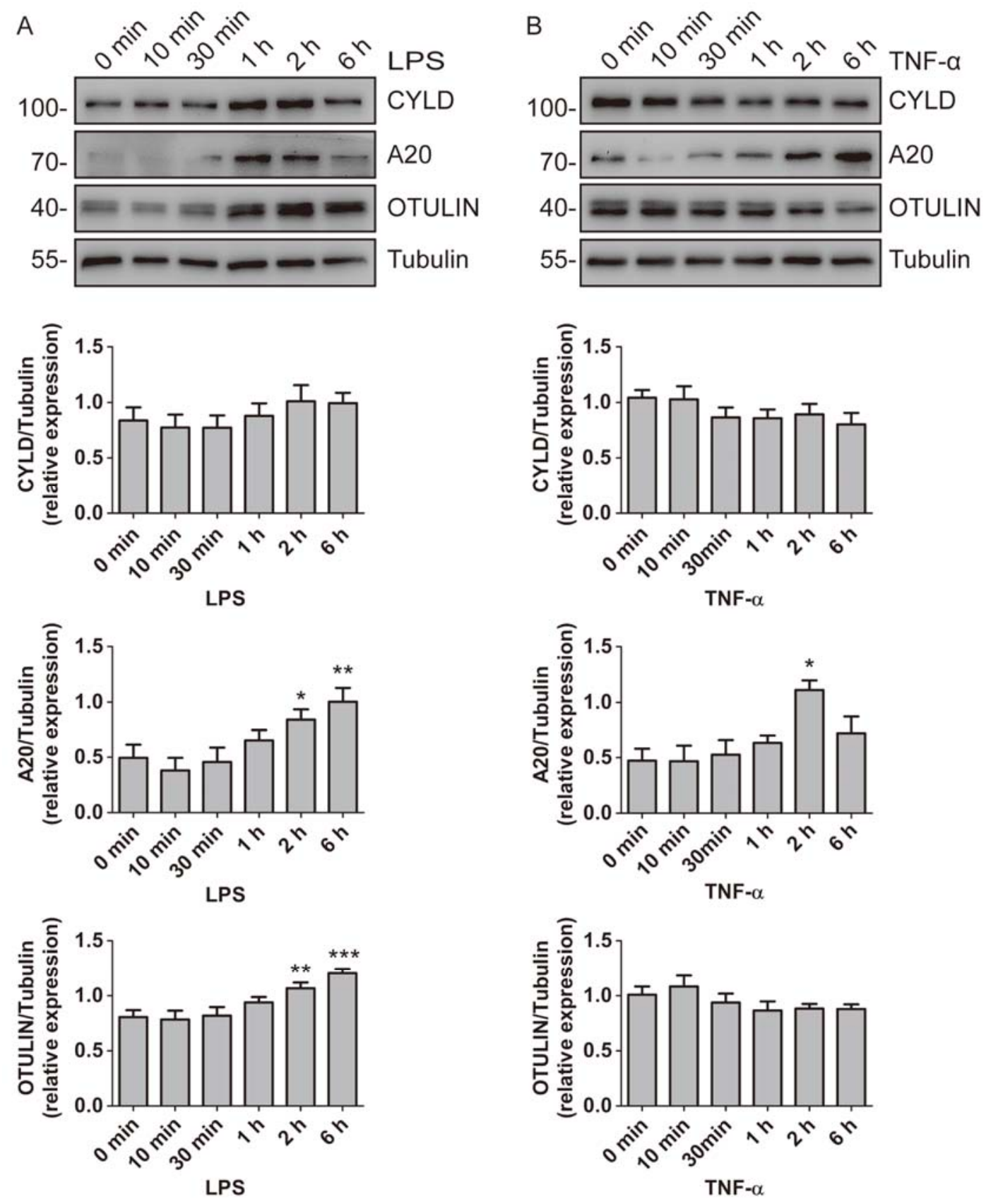

Fig. 4 Protein expression of CYLD, A20 and OTULIN in HGFs pretreated with LPS (a) or TNF- $\alpha(\mathbf{b})$ for the indicated periods of time. Below, quantification of the band intensity results, presented relative to Tubulin. ${ }^{0} P<0.05$, 国 $P<0.01$, 国国 $P<0.001,0 \mathrm{~h}$ versus $2 \mathrm{~h}$ or 6h, one-way ANOVA with Dunnett's multiple comparison test. Data presented are from three independent experiments 


\section{Discussion}

It is known that DUBs such as CYLD, A20 and OTULIN can inhibit inflammatory responses by repressing NF-kB and other signaling pathways $[10,11,25,26]$. In the present study, both biopsies of human gingiva and HGFs show the expression of CYLD, A20 and OTULIN. Furthermore, the expression of CYLD, A20 and OTULIN in HGFs exhibits distinct regulation by different stimuli. Our findings thus imply that CYLD, A20 and OTULIN may play a part in periodontal inflammatory responses.

Previous studies demonstrated that CYLD, A20 and OTULIN are all critical players in suppressing inflammation and immunity $[7,9,26]$. The deficiency of these DUBs may lead to constitutive NF- $\kappa$ B responses, enhance NF- $\kappa B$-dependent gene expression, and result in excessive production of proinflammatory cytokines $[5,8,9]$. In our present study, we characterized the expression of CYLD, A20 and OTULIN in human gingiva and HGFs. In line with our findings, a previous study also confirmed A20 expression in human gingival tissue [27]. Given the role of these DUBs in inhibiting inflammation, we postulate that these DUBs may be involved in the development of periodontitis.

It is known that CYLD expression can be upregulated by many stimuli. In the present study, we found that both LPS and TNF- $\alpha$ did not increase the mRNA level of CYLD. Similarly, western blotting data also showed that the protein level of CYLD had no obvious change in the presence of LPS or TNF- $\alpha$. These findings are in line with our previous study [4]. In contrast, several early reports indicated that CYLD expression was upregulated by LPS or TNF- $\alpha$ treatment [28-30]. The potential mechanisms for this discrepancy remain unknown, but probably due to cell-type-specific differences.

A20, a key regulator of inflammation, is expressed at a low level in most cell types and can be induced by various stimuli including TNF- $\alpha$, LPS, and IL-1 [9]. In this 
study, the mRNA level of A20 was markedly upregulated by TNF- $\alpha$. In contrast, LPS treatment led to a decrease in A20 mRNA expression. As expected, both LPS and TNF$\alpha$ led to the increase of A20 protein expression. We found a lack of concordance between the protein and mRNA levels of A20 in HGFs upon LPS treatment. This result was unexpected but interesting. Many prior works have demonstrated that the expression levels of mRNA and protein are often poorly correlated [31-33]. This discrepancy may be attributed to other types of regulation, such as post-transcriptional processing and protein degradation $[31,32]$. In addition, several recent studies indicated that A20 may act as a critical regulator in the pathogenesis of periodontitis [27, 34-36].

OTULIN was identified as the deubiquitinase that specifically hydrolyzes linear polyubiquitin chains and suppresses NF-אB activation [37]. Recent work suggested that OTULIN can limit inflammation by deubiquitinating LUBAC [10]. However, the regulation of OTULIN is yet to be determined. A recent work showed that porcine reproductive and respiratory syndrome virus infection upregulates the expression of OTULIN [38]. In our current study, LPS resulted in the increase of the mRNA and protein expression of OTULIN. In contrast, the OTULIN mRNA level was decreased upon TNF- $\alpha$ stimulation. Unexpectedly, TNF- $\alpha$ treatment did not change the protein expression of OTULIN. Further studies need to be carried out to investigate the precise mechanism of OTULIN regulation.

\section{Conclusions}

In summary, we show that the DUBs CYLD, A20 and OTULIN are expressed in human gingival tissues and are distinctly regulated by different stimuli. Given the role of these DUBs in limiting inflammation, our findings suggest that CYLD, A20 and OTULIN might be involved in the pathogenesis of periodontitis and might act as potential 
therapeutic targets to treat periodontitis. Therefore, the exact effects of these DUBs on periodontal inflammatory responses require further investigation.

\section{Abbreviations}

DUBs: Deubiquitinating enzymes; HGFs: Human gingival fibroblasts; PBS: Phosphate buffered saline; RT-PCR: Reverse transcription polymerase chain reaction

\section{Acknowledgments}

We are grateful to Yan-Hui Li for her critical reading of the manuscript.

\section{Authors' Contributions}

Yong-Wei Fu is the major person to complete this study. Hong-Zhi Xu is the major person to write the paper.

\section{Funding}

This work was supported by Lianyungang Health Development Project with Science and Education (2017-26).

\section{Availability of data and materials}

The datasets used in the current study are available from the corresponding author on reasonable request.

\section{Ethics approval and consent to participate}

This study was approved by The Ethics Committee of Nanjing Medical University (2018389). Informed consent forms were assigned by all individuals before they were enrolled in the research.

\section{Consent for publication}

Not applicable.

\section{Competing interests}

The authors declare no conflicts of interest. 


\section{References}

1. Ciechanover A: The ubiquitin-proteasome proteolytic pathway. Cell 1994, 79(1):13-21.

2. Goldstein G, Scheid M, Hammerling U, Schlesinger DH, Niall HD, Boyse EA: Isolation of a polypeptide that has lymphocyte-differentiating properties and is probably represented universally in living cells. Proceedings of the National Academy of Sciences of the United States of America 1975, 72(1):11-15.

3. Swatek KN, Komander D: Ubiquitin modifications. Cell research 2016, 26(4):399-422

4. Fu YW, Li L, Wang XQ, Zhou Y, Zhu LF, Mei YM, Xu Y: The inhibitory effect of the deubiquitinase cylindromatosis (CYLD) on inflammatory responses in human gingival fibroblasts. Oral Diseases 2020.

5. Iwai K, Fujita H, Sasaki Y: Linear ubiquitin chains: NF-kappaB signalling, cell death and beyond. Nature reviews Molecular cell biology 2014, 15(8):503-508.

6. Witt A, Vucic D: Diverse ubiquitin linkages regulate RIP kinases-mediated inflammatory and cell death signaling. Cell death and differentiation 2017, 24(7):1160-1171.

7. Aksentijevich I, Zhou Q: NF-kappaB Pathway in Autoinflammatory Diseases: Dysregulation of Protein Modifications by Ubiquitin Defines a New Category of Autoinflammatory Diseases. Frontiers in immunology 2017, 8:399.

8. Zhang Q, Lenardo MJ, Baltimore D: $\mathbf{3 0}$ Years of NF-kappaB: A Blossoming of Relevance to Human Pathobiology. Cell 2017, 168(1-2):37-57.

9. Lork M, Verhelst $K$, Beyaert R: CYLD, A20 and OTULIN deubiquitinases in NF-kappaB signaling and cell death: so similar, yet so different. Cell death and differentiation 2017, 24(7):1172-1183.

10. Heger K, Wickliffe KE, Ndoja A, Zhang J, Murthy A, Dugger DL, Maltzman A, de Sousa EMF, Hung $\mathrm{J}$, Zeng $\mathrm{Y}$ et al: OTULIN limits cell death and inflammation by deubiquitinating LUBAC. Nature 2018, 559(7712):120-124.

11. Ji YX, Huang Z, Yang X, Wang X, Zhao LP, Wang PX, Zhang XJ, Alves-Bezerra $M$, Cai L, Zhang $P$ et al: The deubiquitinating enzyme cylindromatosis mitigates nonalcoholic steatohepatitis. Nature medicine 2018, 24(2):213-223.

12. Polykratis A, Martens A: A20 prevents inflammasome-dependent arthritis by inhibiting macrophage necroptosis through its ZnF7 ubiquitin-binding domain. 2019, 21(6):731-742.

13. Priem D, van Loo G, Bertrand MJM: $\mathbf{A 2 0}$ and Cell Death-driven Inflammation. Trends in immunology 2020, 41(5):421-435.

14. Papapanou PN, Sanz M, Buduneli N, Dietrich T, Feres M, Fine DH, Flemmig TF, Garcia R, Giannobile WV, Graziani F et al: Periodontitis: Consensus report of workgroup 2 of the 2017 World Workshop on the Classification of Periodontal and Peri-Implant Diseases and Conditions. J Periodontol 2018, 89 Suppl 1:S173-s182.

15. Hasturk $\mathrm{H}$, Kantarci $\mathrm{A}$ : Activation and resolution of periodontal inflammation and its systemic impact. Periodontology 2000 2015, 69(1):255-273.

16. Meyle J, Chapple I: Molecular aspects of the pathogenesis of periodontitis. Periodontology 2000 2015, 69(1):7-17.

17. Ebersole JL, Graves CL, Gonzalez OA, Dawson D, 3rd, Morford LA, Huja PE, Hartsfield JK, Jr., Huja SS, Pandruvada S, Wallet SM: Aging, inflammation, immunity and periodontal disease. Periodontology 2000 2016, 72(1):54-75.

18. Cekici A, Kantarci A, Hasturk H, Van Dyke TE: Inflammatory and immune pathways in the pathogenesis of periodontal disease. Periodontology 2000 2014, 64(1):57-80.

19. Van Dyke TE: Commentary: Periodontitis Is Characterized by an Immuno-Inflammatory HostMediated Destruction of Bone and Connective Tissues That Support the Teeth. Journal of Periodontology 2014, 85(4):509-511.

20. Bartold PM, Van Dyke TE: Host modulation: controlling the inflammation to control the infection. Periodontology 2000 2017, 75(1):317-329.

21. Taniguchi K, Karin M: NF-kappaB, inflammation, immunity and cancer: coming of age. Nature reviews Immunology 2018, 18(5):309-324.

22. Ambili R, Janam P, Saneesh Babu PS, Prasad M, Vinod D, Anil Kumar PR, Kumary TV, Asha Nair S: Differential expression of transcription factors NF-kappaB and STAT3 in periodontal ligament fibroblasts and gingiva of healthy and diseased individuals. Archives of oral biology 2017, 82:19-26. 
23. Garcia-Carbonell R, Wong J, Kim JY, Close LA, Boland BS, Wong TL, Harris PA, Ho SB, Das S, Ernst $\mathrm{PB}$ et al: Elevated $\mathbf{A 2 0}$ promotes TNF-induced and RIPK1-dependent intestinal epithelial cell death. Proceedings of the National Academy of Sciences of the United States of America 2018, 115(39):E9192-E9200.

24. Golz L, Memmert S, Rath-Deschner B, Jager A, Appel T, Baumgarten G, Gotz W, Frede S: Hypoxia and P. gingivalis synergistically induce HIF-1 and NF-kappaB activation in PDL cells and periodontal diseases. Mediators of inflammation 2015, 2015:438085.

25. Malynn BA, Ma A: A20: A multifunctional tool for regulating immunity and preventing disease. Cellular immunology 2019, 340:103914.

26. Wertz IE, Newton K, Seshasayee D, Kusam S, Lam C, Zhang J, Popovych N, Helgason E, Schoeffler $\mathrm{A}$, Jeet $\mathrm{S}$ et al: Phosphorylation and linear ubiquitin direct A20 inhibition of inflammation. Nature 2015, 528(7582):370-375.

27. Crump KE, Oakley JC, Xia-Juan X, Madu TC, Devaki S, Mooney EC, Sahingur SE: Interplay of TollLike Receptor 9, Myeloid Cells, and Deubiquitinase A20 in Periodontal Inflammation. Infection and immunity 2017, 85(1).

28. Jono H, Lim JH, Chen LF, Xu H, Trompouki E, Pan ZK, Mosialos G, Li JD: NF-kappaB is essential for induction of CYLD, the negative regulator of NF-kappaB: evidence for a novel inducible autoregulatory feedback pathway. The Journal of biological chemistry 2004, 279(35):3617136174.

29. Fukuda M, Fukuda F, Horiuchi Y, Oku Y, Suzuki S, Kusama K, Sakashita H: Expression of CYLD, NFkappaB and NF-kappaB-related factors in salivary gland tumors. In vivo (Athens, Greece) 2006, 20(4):467-472.

30. Bikker R, Christmann M, Preuß K, Welz B, Friesenhagen J, Dittrich-Breiholz O, Huber R, Brand K: TNF phase III signalling in tolerant cells is tightly controlled by A20 and CYLD. Cellular signalling 2017, 37:123-135.

31. Chen G, Gharib TG, Huang CC, Taylor JM, Misek DE, Kardia SL, Giordano TJ, lannettoni MD, Orringer $\mathrm{MB}$, Hanash $\mathrm{SM}$ et al: Discordant protein and mRNA expression in lung adenocarcinomas. Molecular \& cellular proteomics : MCP 2002, 1(4):304-313.

32. de Sousa Abreu R, Penalva LO, Marcotte EM, Vogel C: Global signatures of protein and mRNA expression levels. Molecular biosystems 2009, 5(12):1512-1526.

33. Griffin TJ, Gygi SP, Ideker T, Rist B, Eng J, Hood L, Aebersold R: Complementary profiling of gene expression at the transcriptome and proteome levels in Saccharomyces cerevisiae. Molecular \& cellular proteomics : MCP 2002, 1(4):323-333.

34. Hong JY, Bae WJ, Yi JK, Kim GT, Kim EC: Anti-inflammatory and anti-osteoclastogenic effects of zinc finger protein A20 overexpression in human periodontal ligament cells. Journal of periodontal research 2016, 51(4):529-539.

35. Li Y, Mooney EC, Holden SE, Xia XJ, Cohen DJ, Walsh SW, Ma A, Sahingur SE: A20 Orchestrates Inflammatory Response in the Oral Mucosa through Restraining NF-kappaB Activity. Journal of immunology 2019, 202(7):2044-2056.

36. Mooney EC, Sahingur SE: The Ubiquitin System and A20: Implications in Health and Disease. Journal of dental research 2021, 100(1):10-20.

37. Keusekotten K, Elliott PR, Glockner L, Fiil BK, Damgaard RB, Kulathu Y, Wauer T, Hospenthal MK, Gyrd-Hansen M, Krappmann D et al: OTULIN antagonizes LUBAC signaling by specifically hydrolyzing Met1-linked polyubiquitin. Cell 2013, 153(6):1312-1326.

38. Su Y, Shi P, Zhang L, Lu D, Zhao C, Li R, Zhang L, Huang J: The Superimposed Deubiquitination Effect of OTULIN and Porcine Reproductive and Respiratory Syndrome Virus (PRRSV) Nsp11 Promotes Multiplication of PRRSV. Journal of virology 2018, 92(9).

Figure legends 
Fig. 1 Immunohistochemistry for CYLD, A20 and OTULIN on sections from gingival tissue. a The negative control of immunohistochemistry. b CYLD staining in gingival tissue. Arrows show positive staining for CYLD. c A20 staining in gingival tissue. Arrows show positive staining for A20. d OTULIN staining in gingival tissue. Arrows show positive staining for OTULIN. Scale bar, $50 \mu \mathrm{m}$

Fig. 2 Immunofluorescence staining for CYLD (a), A20 (b) and OTULIN (c) in HGFs. Scale bar, $50 \mu \mathrm{m}$

Fig. 3 mRNA levels of CYLD, A20 and OTULIN in HGFs pretreated with LPS (a, b, c) or TNF- $\square(\mathbf{d}, \mathbf{e}, \mathbf{f})$ for the indicated periods of time. ${ }^{\square} P<0.05$, ${ }^{0} P<0.01$, ${ }^{0}{ }^{0} P<0.001,0$ $\mathrm{h}$ versus $1 \mathrm{~h}, 2 \mathrm{~h}, 3 \mathrm{~h}, 4 \mathrm{~h}$ or $5 \mathrm{~h}$, one-way ANOVA with Dunnett's multiple comparison test. Data presented are from three independent experiments

Fig. 4 Protein expression of CYLD, A20 and OTULIN in HGFs pretreated with LPS (a) or TNF- $\alpha$ (b) for the indicated periods of time. Below, quantification of the band intensity results, presented relative to Tubulin. ${ }^{\square} P<0.05$, ${ }^{0} P<0.01$, 国回 $P<0.001,0 \mathrm{~h}$ versus $2 \mathrm{~h}$ or $6 \mathrm{~h}$, one-way ANOVA with Dunnett's multiple comparison test. Data presented are from three independent experiments 